\title{
Hybrid 193 nm Ultraviolet Photodissociation Mass Spectrometry Localizes Cardiolipin Unsaturations
}

\author{
Luis A. Macias, Clara L. Feider, Livia S. Eberlin, Jennifer S. Brodbelt ${ }^{*}$ \\ Department of Chemistry, University of Texas, Austin, TX 78712 \\ Correspondence: jbrodbelt@cm.utexas.edu
}

\section{Supporting Information}

Table of Contents

\begin{tabular}{|c|c|c|}
\hline & Content & Page no. \\
\hline 1 & Experimental Methods & S-2 \\
\hline 2 & Table S1. Structures of all standard cardiolipins (CLs) & S3 \\
\hline 3 & Table S2. CLs identified in E. coli extract & S-4 \\
\hline 4 & Table S3. CLs identified in human papillary thyroid carcinoma extract & S-5 \\
\hline 5 & Scheme S1. Structural composition of CLs & S-6 \\
\hline 6 & Scheme S2. Carbon numbering for fatty acids. & S-7 \\
\hline 7 & Figure S1. Negative mode CL MS ${ }^{1}$ & S-8 \\
\hline 8 & Figure S2. CID of singly deprotonated CL & S-9 \\
\hline 9 & Figure S3. UVPD of singly deprotonated CL & S-10 \\
\hline 10 & Figure S4. Zoomed view of Figure 1B & S-11 \\
\hline 11 & $\begin{array}{l}\text { Figure S5. Negative mode } \mathrm{MS}^{1} \text { spectrum of papillary thyroid } \\
\text { carcinoma total lipid extract. }\end{array}$ & S-12 \\
\hline 12 & $\begin{array}{c}\text { Figure S6. CID and CID/UVPD of CL } \\
(16: 0 / 18: 1(9 \mathrm{Z})) /(16: 0 / 18: 1(9 \mathrm{Z}))\end{array}$ & S-13 \\
\hline 13 & $\begin{array}{l}\text { Figure S7. HCD and HCD/UVPD of CL } \\
(16: 0 / 18: 1(9 \mathrm{Z}) /(16: 0 / 18: 1(9 \mathrm{Z}))\end{array}$ & S-14 \\
\hline 14 & $\begin{array}{l}\text { Figure S8. CID of doubly deprotonated CL } \\
(22: 1(13 Z) / 22: 1(13 Z)) /(22: 1(13 Z) / 14: 1(9 Z)\end{array}$ & S-15 \\
\hline 15 & $\begin{array}{c}\text { Figure S9. CID of } \mathrm{m} / \mathrm{z} 711.48 \text { and } \mathrm{m} / \mathrm{z} 725.49 \text { from a papillary thyroid } \\
\text { carcinoma lipid extract }\end{array}$ & S-16 \\
\hline 16 & References & S-17 \\
\hline
\end{tabular}




\section{Experimental}

Nomenclature. Lipid shorthand notation, as described by Liebsich et al., ${ }^{1}$ was adopted. Briefly, the lipid subclass is indicated by letter abbreviations (i.e. CL for cardiolipins) with the acyl composition indicated by the number of carbons in the acyl chain followed by a colon and the degree of unsaturation. When the sn-stereochemistry is known, the acyl chain composition at the sn-1 position is followed by a "/" and the sn-2 composition. When the sn-stereochemistry is unknown, the acyl chain compositions are separated by "_." If the site of unsaturation is known, the degree of unsaturation is followed by the carbon number of the unsaturation position. The geometry of the unsaturation is indicated by $\mathrm{Z}, \mathrm{E}$, or $\Delta$, corresponding to a cis, trans, or unknown conformation, respectively. For example, PA 16:0/18:1(9Z) indicates a phosphatidic acid with palmitate at the $s n-1$ position and oleate at the $s n-2$ position. The use of "/" is also appropriate when discussing phospholipid standards of known sn regiochemistry, or when discussing structures that incorporate the same fatty acid at both the $s n-1$ and $s n-2$ positions, such as PA 16:0/16:0. The shorthand notation for CL was modified to indicate that the acyl chain composition at each PA moiety is known. For example, CL (14:0_16:0)_(18:0_20:0) indicates that even though the acyl chain compositions cannot be designated to the sn-1, sn-2, sn-1', or sn-2' positions, it is known that one constituent PA moiety is composed of a 14:0 and a 16:0 acyl chain, while the other moiety is composed a 18:0 and a 20:0 acyl chain. CL (14:0_16:0)/(18:0_20:0) indicates that it is known that PA 14:0_16:0 is at the 1' position of the central glycerol and that PA 18:0_20:0 is at the 3' position of the central glycerol. Cardiolipins are often described as heteroacylated, Heteroacylated signifies that all four of the acyl chains are not the same. In contrast, the term "homo-acylated" is used to describe cardiolipins that are composed of four identical fatty acids as a result of acyl chain remodeling. The term heteroacylated is used refer to variations in double bond positions as well as chain lengths. The nomenclature was also modified to describe cyclopropyl fatty acids as previously described. ${ }^{2}$ 
Table S1: Structures of all standard cardiolipins

(Dipid Name


Table S2: Identified CLs in E. coli extract

\begin{tabular}{|c|c|c|c|c|c|c|c|c|}
\hline \multirow{3}{*}{$\begin{array}{l}\text { MS2 } \\
\text { Precursor } \\
(\mathrm{m} / \mathrm{z})\end{array}$} & \multicolumn{4}{|c|}{ CID } & \multirow{2}{*}{\multicolumn{3}{|c|}{$\frac{\text { CID/UVPD }}{\text { Fatty Acid Characterization }}$}} & \multirow{3}{*}{ Cardiolipin } \\
\hline & \multicolumn{2}{|c|}{$\begin{array}{l}\text { Fatty Acid } \\
\text { Assignment }\end{array}$} & \multicolumn{2}{|c|}{ PA Assignment } & & & & \\
\hline & $m / z$ & $\begin{array}{l}\text { Fatty } \\
\text { Acid }\end{array}$ & $m / z$ & $\begin{array}{c}\text { PA } \\
\text { Moiety }\end{array}$ & $\begin{array}{c}\text { MS3 } \\
\text { Precursor } \\
(\mathrm{m} / \mathrm{z})\end{array}$ & $\begin{array}{l}\text { Unsaturation Diagnostic } \\
\text { Ions }(m / z)\end{array}$ & $\begin{array}{l}\text { Unsaturation } \\
\text { Assignment }\end{array}$ & \\
\hline 659.45 & \begin{tabular}{|l|}
1091.68 \\
1065.69 \\
1063.66 \\
\end{tabular} & $\begin{array}{l}14: 0 \\
16: 1 \\
16: 0\end{array}$ & $\begin{array}{l}701.47 \\
673.44\end{array}$ & $\begin{array}{l}16: 1 \_16: 0 \\
16: 1 \_14: 0\end{array}$ & 1065.68 & $955.56,979.57$ & $16: 1(9 \Delta)$ & 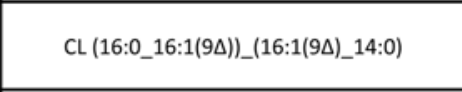 \\
\hline 672.46 & \begin{tabular}{|l|}
1117.71 \\
1091.69 \\
1089.69 \\
1063.66 \\
\end{tabular} & $\begin{array}{l}14: 0 \\
16: 1 \\
16: 0 \\
18: 1 \\
\end{array}$ & $\begin{array}{l}727.49 \\
701.48 \\
699.46 \\
672.45 \\
\end{array}$ & $\begin{array}{l}\text { 18:1_16:1 } \\
\text { 16:1_16:0 } \\
\text { 16:1/16:1 } \\
16: 1 \_14: 0 \\
\end{array}$ & 1091.69 & $981.57,1005.58$ & $\begin{array}{l}16: 1(9 \Delta) \\
18: 1(11 \Delta)\end{array}$ & 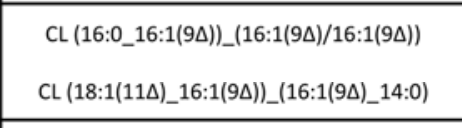 \\
\hline 673.47 & \begin{tabular}{|c|}
1093.71 \\
1091.7 \\
\end{tabular} & $\begin{array}{l}16: 1 \\
16: 0 \\
\end{array}$ & 701.48 & 16:1_16:0 & 1093.71 & $983.59,1007.59$ & $16: 1(9 \Delta)$ & CL $\left(16: 0 \_16: 1(9 \Delta)\right) /\left(16: 0 \_16: 1(9 \Delta)\right)$ \\
\hline 680.47 & \begin{tabular}{|l|}
1107.72 \\
1105.71 \\
1093.71 \\
\end{tabular} & $\begin{array}{l}16: 1 \\
16: 0 \\
17: 1 \\
\end{array}$ & $\begin{array}{l}715.49 \\
701.47\end{array}$ & $\begin{array}{l}17: 1 \_16: 0 \\
16: 1 \_16: 0\end{array}$ & $\begin{array}{l}1107.72 \\
1093.71\end{array}$ & $\begin{array}{l}995.59,1009.61 \\
983.59,1007.59\end{array}$ & $\begin{array}{l}17: 1(\mathrm{c} 9 \Delta) \\
16: 1(9 \Delta)\end{array}$ & 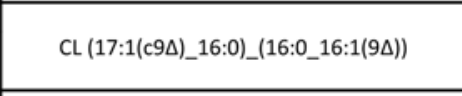 \\
\hline 679.46 & \begin{tabular}{c|}
1117.7 \\
1105.71 \\
1103.69 \\
1091.7 \\
1077.67
\end{tabular} & $\begin{array}{l}15: 0 \\
16: 1 \\
16: 0 \\
17: 1 \\
18: 1\end{array}$ & $\begin{array}{l}727.49 \\
715.49 \\
713.48 \\
701.48 \\
699.46 \\
687.46 \\
\end{array}$ & $\begin{array}{l}18: 1 \_16: 1 \\
17: 1 \_16: 0 \\
17: 1 \_16: 1 \\
16: 1 \_16: 0 \\
16: 1 / 16: 1 \\
16: 1 \_15: 0 \\
\end{array}$ & 1091.69 & $995.59,1019.59$ & $\begin{array}{c}18: 1(11 \Delta) \\
16: 1(9 \Delta) \\
17: 1(c 9 \Delta) \\
16: 1(9 \Delta)\end{array}$ & 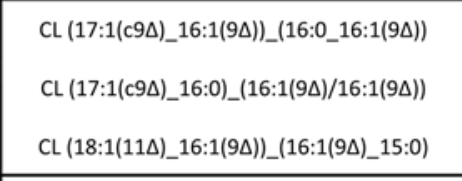 \\
\hline 685.47 & \begin{tabular}{|l|}
1117.71 \\
1089.68 \\
\end{tabular} & \begin{tabular}{|l|}
$16: 1$ \\
$18: 1$ \\
\end{tabular} & $\begin{array}{l}727.49 \\
699.46 \\
\end{array}$ & \begin{tabular}{|l|}
$16: 1 / 16: 1$ \\
$16: 1 \_18: 1$ \\
\end{tabular} & 1117.71 & $1007.59,1031.59$ & $\begin{array}{c}16: 1(9 \Delta) \\
18: 1(11 \Delta) \\
\end{array}$ & CL $\left(18: 1(11 \Delta) \_16: 1(9 \Delta)\right) /(16: 1(9 \Delta) / 16: 1(9 \Delta))$ \\
\hline 686.47 & $\begin{array}{l}1119.72 \\
1117.71 \\
1093.69 \\
\end{array}$ & $\begin{array}{l}16: 1 \\
16: 0 \\
18: 1 \\
\end{array}$ & $\begin{array}{l}727.49 \\
701.47\end{array}$ & $\begin{array}{l}\text { 18:1_16:1 } \\
16: 0 \_16: 1\end{array}$ & 1119.72 & $1009.61,1033.61$ & $\begin{array}{c}16: 1(9 \Delta) \\
18: 1(11 \Delta)\end{array}$ & 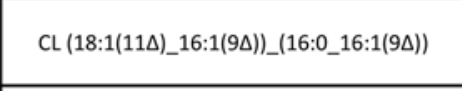 \\
\hline 687.48 & \begin{tabular}{|l|}
1121.74 \\
1119.72 \\
1093.71 \\
\end{tabular} & $\begin{array}{l}16: 1 \\
16: 0 \\
18: 1 \\
\end{array}$ & $\begin{array}{l}701.47 \\
729.51\end{array}$ & $\begin{array}{l}\text { 16:0_16:1 } \\
\text { 18:1_16:0 }\end{array}$ & 1093.71 & $1001.62,1035.62$ & $\begin{array}{l}18: 1(11 \Delta) \\
16: 1(9 \Delta)\end{array}$ & 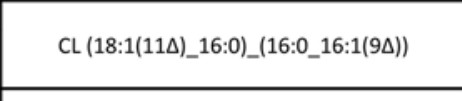 \\
\hline & \begin{tabular}{|l|}
1131.73 \\
1117.71
\end{tabular} & $\begin{array}{l}16: 1 \\
17: 1\end{array}$ & $\begin{array}{l}741.51 \\
727.49\end{array}$ & $\begin{array}{l}\text { 18:1_17:1 } \\
\text { 17:1_16:1 }\end{array}$ & 1131.73 & $1021.61,1045.61$ & $\begin{array}{c}18: 1(11 \Delta) \\
16: 1(9 \Delta)\end{array}$ & 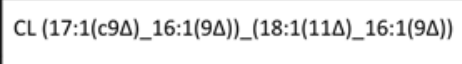 \\
\hline 692.47 & 1103.7 & $18: 1$ & $\begin{array}{l}713.48 \\
699.46\end{array}$ & $\begin{array}{l}16: 1 \_18: 1 \\
16: 1 / 16: 1\end{array}$ & 1117.71 & $1007.60,1031.60$ & $\begin{array}{c}17: 1(c 9 \Delta) \\
18: 1(11 \Delta) \\
16: 1(9 \Delta) \\
\end{array}$ & CL (18:1(11 $\left.\Delta) \_17: 1(c 9 \Delta)\right) \_(16: 1(9 \Delta) / 16: 1(9 \Delta))$ \\
\hline 693.47 & $\begin{array}{l}1133.74 \\
1131.73 \\
1119.72 \\
1105.71\end{array}$ & $\begin{array}{l}16: 1 \\
16: 0 \\
17: 1 \\
18: 1\end{array}$ & $\begin{array}{l}727.49 \\
715.49\end{array}$ & $\begin{array}{l}\text { 18:1_16:1 } \\
17: 1 \_16: 0\end{array}$ & 1133.74 & $\begin{array}{l}1023.62,1047.62 \\
1021.61,1035.62 \\
1009.61,1033.61 \\
\end{array}$ & $\begin{array}{c}18: 1(11 \Delta) \\
16: 1(9 \Delta) \\
17: 1(c 9 \Delta) \\
18: 1(11 \Delta) \\
16: 1(9 \Delta) \\
\end{array}$ & 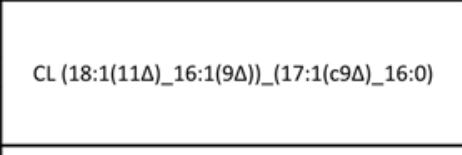 \\
\hline & $\begin{array}{l}1135.75 \\
1133.73\end{array}$ & $\begin{array}{l}16: 1 \\
16: 0\end{array}$ & $\begin{array}{l}743.52 \\
729.51\end{array}$ & $\begin{array}{l}\text { 19:1_16:0 } \\
\text { 18:1_16:0 }\end{array}$ & $\begin{array}{l}1135.75 \\
1121.74 \\
\end{array}$ & $\begin{array}{l}1023.62,1037.63 \\
1011.62,1035.62 \\
\end{array}$ & $\begin{array}{c}19: 1(\mathrm{c} 11 \Delta) \\
18: 1(11 \Delta)\end{array}$ & 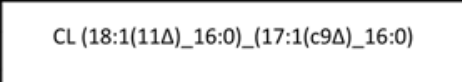 \\
\hline 694.48 & \begin{tabular}{|l|}
1121.74 \\
1107.73 \\
1093.71 \\
\end{tabular} & $\begin{array}{l}17: 1 \\
18: 1 \\
19: 1 \\
\end{array}$ & $\begin{array}{l}715.49 \\
701.48\end{array}$ & $\begin{array}{l}\text { 17:1_16:0 } \\
16: 1 \_16: 0\end{array}$ & $\begin{array}{l}1107.73 \\
1093.71\end{array}$ & $\begin{array}{l}995.59,1009.61 \\
983.59,1007.60\end{array}$ & $\begin{array}{l}17: 1(c 9 \Delta) \\
16: 1(9 \Delta)\end{array}$ & CL (19:1(c11 $\left.) \_16: 0\right) \_\left(16: 0 \_16: 1(9 \Delta)\right)$ \\
\hline 699.48 & \begin{tabular}{|l|}
1147.75 \\
1117.71
\end{tabular} & $\begin{array}{l}16: 1 \\
18: 1\end{array}$ & $\begin{array}{l}755.52 \\
727.49 \\
699.48 \\
\end{array}$ & \begin{tabular}{l|}
$18: 1 / 18: 1$ \\
$18: 1 \_16: 1$ \\
$16: 1 / 16: 1$ \\
\end{tabular} & 1147.75 & $1035.62,1059.62$ & $\begin{array}{l}18: 1(11 \Delta) \\
16: 1(9 \Delta)\end{array}$ & 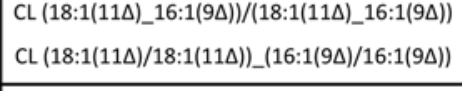 \\
\hline 700.48 & $\begin{array}{l}1147.75 \\
1145.74 \\
1119.72\end{array}$ & $\begin{array}{l}16: 1 \\
16: 0 \\
18: 1\end{array}$ & $\begin{array}{l}755.52 \\
729.51 \\
727.49 \\
701.48 \\
\end{array}$ & $\begin{array}{l}18: 1 / 18: 1 \\
18: 1 \_16: 0 \\
18: 1 \_16: 1 \\
16: 1 \_16: 0\end{array}$ & 1119.72 & $1009.61,1033.61$ & $\begin{array}{c}18: 1(11 \Delta) \\
16: 1(9 \Delta)\end{array}$ & 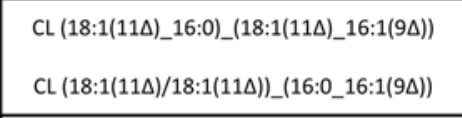 \\
\hline & $\begin{array}{l}1159.76 \\
1145.74\end{array}$ & $\begin{array}{l}16: 1 \\
17: 1\end{array}$ & $\begin{array}{l}755.52 \\
741.51\end{array}$ & $\begin{array}{l}18: 1 / 18: 1 \\
18: 1 \_17: 1\end{array}$ & 1131.73 & $1021.62,1045.62$ & $\begin{array}{c}18: 1(11 \Delta) \\
16: 1(9 \Delta)\end{array}$ & CL (18:1(11 11$\left.) \_17: 1(c 9 \Delta)\right) \_\left(18: 1(11 \Delta) \_16: 1(9 \Delta)\right)$ \\
\hline 706.48 & 1131.73 & $18: 1$ & $\begin{array}{l}727.49 \\
713.48\end{array}$ & $\begin{array}{l}18: 1 \_16: 1 \\
17: 1 \_16: 1\end{array}$ & 1145.74 & $1035.63,1059.63$ & $\begin{array}{c}17: 1(c 9 \Delta) \\
18: 1(11 \Delta) \\
16: 1(9 \Delta) \\
\end{array}$ & CL (18:1(11 $\Delta) / 18: 1(11 \Delta) \_\left(17: 1(c 9 \Delta) \_16: 1(9 \Delta)\right)$ \\
\hline 713.49 & \begin{tabular}{|l|}
1145.74 \\
1173.77 \\
\end{tabular} & $\begin{array}{l}18: 1 \\
16: 1 \\
\end{array}$ & $\begin{array}{l}755.52 \\
727.49 \\
\end{array}$ & $\begin{array}{l}18: 1 / 18: 1 \\
18: 1 \_16: 1 \\
\end{array}$ & 1145.74 & $1035.63,1059.63$ & $\begin{array}{c}18: 1(11 \Delta) \\
16: 1(9 \Delta) \\
\end{array}$ & CL $(18: 1(11 \Delta) / 18: 1(11 \Delta)) \_\left(18: 1(11 \Delta) \_16: 1(9 \Delta)\right)$ \\
\hline 714.5 & \begin{tabular}{|l|}
1173.77 \\
1147.75 \\
\end{tabular} & $\begin{array}{l}16: 0 \\
18: 1 \\
\end{array}$ & $\begin{array}{l}755.52 \\
729.51 \\
\end{array}$ & $\begin{array}{l}18: 1 / 18: 1 \\
18: 1 \quad 16: 0\end{array}$ & 1147.75 & $1037.64,1061.64$ & $18: 1(11 \Delta)$ & 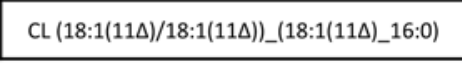 \\
\hline
\end{tabular}


Table S3: Identified CLs in human papillary thyroid carcinoma extract

\begin{tabular}{|c|c|c|c|c|c|c|c|c|}
\hline \multirow{3}{*}{$\begin{array}{l}\text { MS2 } \\
\text { Precursor } \\
(\mathrm{m} / \mathrm{z})\end{array}$} & \multicolumn{4}{|c|}{ CID } & \multirow{2}{*}{\multicolumn{3}{|c|}{$\begin{array}{c}\text { CID/UVPD } \\
\text { Fatty Acid Characterization }\end{array}$}} & \multirow[b]{3}{*}{ Cardiolipin } \\
\hline & \multicolumn{2}{|c|}{$\begin{array}{l}\text { Fatty Acid } \\
\text { Assignment }\end{array}$} & \multicolumn{2}{|c|}{ PA Assignment } & & & & \\
\hline & $m / z$ & $\begin{array}{l}\text { Fatty } \\
\text { Acid }\end{array}$ & $m / z$ & $\begin{array}{c}\text { PA } \\
\text { Moiety }\end{array}$ & $\begin{array}{l}\text { MS3 } \\
\text { Precursor } \\
(\mathrm{m} / \mathrm{z})\end{array}$ & $\begin{array}{c}\text { Unsaturation } \\
\text { Diagnostic Pairs } \\
(\mathrm{m} / \mathrm{z})\end{array}$ & $\begin{array}{l}\text { Unsaturation } \\
\text { Assignment }\end{array}$ & \\
\hline \multirow{3}{*}{710.47} & \multirow[t]{3}{*}{$\begin{array}{c}1167.72 \\
1141.7\end{array}$} & \multirow[t]{3}{*}{$\begin{array}{l}16: 1 \\
18: 2\end{array}$} & \multirow[t]{3}{*}{$\begin{array}{l}725.48 \\
751.49\end{array}$} & \multirow[t]{3}{*}{$\begin{array}{l}18: 2 \_16: 1 \\
18: 2 / 18: 2\end{array}$} & 1167.72 & $\begin{array}{l}1031.60,1055.59 \\
1071.62,1095.62\end{array}$ & $18: 2(9 \Delta, 12 \Delta)$ & \multirow{3}{*}{$\begin{array}{c}C L \\
(18: 2(9 \Delta, 12 \Delta) / 18: 2(9 \Delta, 12 \Delta)) \_(18: 2(9 \\
\left.\Delta, 12 \Delta) \_16: 1(9 \Delta)\right)\end{array}$} \\
\hline & & & & & \multirow[t]{2}{*}{1141.7} & $\begin{array}{l}1005.58,1029.58 \\
1045.61,1069.61\end{array}$ & \multirow[t]{2}{*}{$18: 2(9 \Delta, 12 \Delta)$} & \\
\hline & & & & & & $1031.59,1055.59$ & & \\
\hline \multirow{3}{*}{713.49} & \multirow{3}{*}{$\begin{array}{l}1171.75 \\
1147.75 \\
1145.73\end{array}$} & \multirow{3}{*}{$\begin{array}{l}16: 0 \\
18: 2 \\
18: 1\end{array}$} & \multirow{3}{*}{$\begin{array}{l}753.51 \\
729.51\end{array}$} & \multirow{3}{*}{$\begin{array}{l}18: 1 \_18: 2 \\
18: 1 \_16: 0\end{array}$} & 1147.75 & $1037.64,1061.64$ & & \multirow{3}{*}{$\begin{array}{c}\mathrm{CL} \\
\left(18: 1(11 \Delta) \_18: 2(9 \Delta, 12 \Delta)\right) \_(18: 1(11 \Delta) \\
\left.\_16: 0\right)\end{array}$} \\
\hline & & & & & \multirow[t]{2}{*}{1145.73} & $\begin{array}{l}1009.61,1033.61 \\
1049.64,1073.64\end{array}$ & \multirow[t]{2}{*}{$18: 2(9 \Delta, 12 \Delta)$} & \\
\hline & & & & & & $1035.62,1059.62$ & & \\
\hline 723.48 & 1167.72 & $18: 2$ & 751.49 & $18: 2 / 18: 2$ & 1167.72 & $\begin{array}{l}1031.59,1055.60 \\
1071.63,1095.63\end{array}$ & $18: 2(9 \Delta, 12 \Delta)$ & $\begin{array}{c}\mathrm{CL} \\
(18: 2(9 \Delta, 12 \Delta) / 18: 2(9 \Delta, 12 \Delta)) /(18: 2(9 \\
\Delta, 12 \Delta) / 18: 2(9 \Delta, 11 \Delta))\end{array}$ \\
\hline & $\begin{array}{l}1169.73 \\
1167.72\end{array}$ & $\begin{array}{l}18: 2 \\
18: 1\end{array}$ & $\begin{array}{l}753.51 \\
751.49\end{array}$ & \begin{tabular}{|l|}
$18: 1 \_18: 2$ \\
$18: 2 / 18: 2$
\end{tabular} & 1169.73 & $\begin{array}{l}1033.61,1057.61 \\
1073.64,1097.64\end{array}$ & $18: 2(9 \Delta, 12 \Delta)$ & $\mathrm{CL}$ \\
\hline 724.49 & & & & & & $1059.62,1083.63$ & $18: 1(11 \Delta)$ & $\left(18: 1(11 \Delta) \_18: 2(9 \Delta, 12 \Delta)\right) \_(18: 2(9 \Delta, 1$ \\
\hline & & & & & 1167.72 & $\begin{array}{l}1031.60,1055.60 \\
1071.63,1095.63\end{array}$ & $18: 2(9 \Delta, 12 \Delta)$ & $2 \Delta) / 18: 2(9 \Delta, 12 \Delta))$ \\
\hline & $\begin{array}{l}1191.72 \\
1167.72\end{array}$ & $\begin{array}{l}18: 2 \\
20: 4\end{array}$ & $\begin{array}{l}775.49 \\
751.49\end{array}$ & \begin{tabular}{|l|}
$20: 4 / 18: 2$ \\
$18: 2 / 18: 2$
\end{tabular} & 1167.72 & $\begin{array}{l}1031.59,1055.59 \\
1071.63,1095.62\end{array}$ & $18: 2(9 \Delta, 12 \Delta)$ & \\
\hline 735.48 & & & & & 1191.72 & $\begin{array}{c}1095.63,1119.63 \\
1055.59,1079.59 \\
1015.57,1039.56 \\
975.53,999.53\end{array}$ & $\begin{array}{c}20: 4(5 \Delta, 8 \Delta, 11 \Delta, 1 \\
4 \Delta) \\
18: 2(9 \Delta, 12 \Delta)\end{array}$ & $\begin{array}{c}\mathrm{CL} \\
\left(20: 4(5 \Delta, 8 \Delta, 11 \Delta, 14 \Delta) \_18: 2(9 \Delta, 12 \Delta)\right) \\
\quad(18: 2(9 \Delta, 12 \Delta) / 18: 2(9 \Delta, 12 \Delta))\end{array}$ \\
\hline & $\begin{array}{l}1193.73 \\
1167.72\end{array}$ & $\begin{array}{l}18: 2 \\
20: 3\end{array}$ & $\begin{array}{l}777.51 \\
751.49\end{array}$ & $\begin{array}{l}20: 3 \_18: 2 \\
18: 2 / 18: 2\end{array}$ & 1167.72 & $\begin{array}{l}1031.59,1055.59 \\
1071.62,1095.62\end{array}$ & $18: 2(9 \Delta, 12 \Delta)$ & $\mathrm{CL}$ \\
\hline 736.49 & & & & & 1193.73 & $\begin{array}{l}1097.64,1121.64 \\
1057.61,1081.61 \\
1017.58,1041.58\end{array}$ & $\begin{array}{c}20: 3(8 \Delta, 11 \Delta, 14 \Delta) \\
18: 2(9 \Delta, 12 \Delta)\end{array}$ & $\begin{array}{c}\left(20: 3(8 \Delta, 11 \Delta, 14 \Delta) \_18: 2(9 \Delta, 12 \Delta)\right) \_(1 \\
8: 2(9 \Delta, 12 \Delta) / 18: 2(9 \Delta, 12 \Delta))\end{array}$ \\
\hline & 1195.75 & $18: 2$ & 779.52 & 20:2_18:2 & & $1031.59,1055.59$ & & \\
\hline 73754 & 1167.72 & $20: 2$ & 751.49 & $18: 2 / 18: 2$ & & $1071.62,1095.62$ & & \\
\hline 137.54 & & & & & 1195.75 & $\begin{array}{l}1059.62,1083.62 \\
1099.65,1123.66\end{array}$ & $\begin{array}{c}20: 2(11 \Delta, 14 \Delta) \\
18: 2(9 \Delta, 12 \Delta)\end{array}$ & 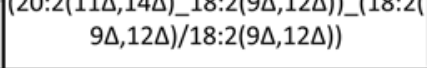 \\
\hline & 1197.76 & $18: 2$ & 779.52 & $20: 2 \_18: 2$ & 1195.75 & $1059.63,1083.63$ & $18: 2(9 \Delta, 12 \Delta)$ & \\
\hline & 1195.75 & $18: 1$ & 753.51 & 18:1_18:2 & 1195.15 & $1099.66,1123.66$ & $20: 2(11 \Delta, 14 \Delta)$ & $\mathrm{CL}$ \\
\hline 738.5 & 1169.73 & $20: 2$ & & & & $1061.64,1085.64$ & $18: 2(9 \Delta, 12 \Delta)$ & $\left(20: 2(11 \Delta, 14 \Delta) \_18: 2(9 \Delta, 12 \Delta)\right) \_(18: 1 \text { ( }$ \\
\hline & & & & & 1197.76 & $1101.67,1125.67$ & $20: 2(11 \Delta, 14 \Delta)$ & 11 $\left.\Delta) \_18: 2(9 \Delta, 12 \Delta)\right)$ \\
\hline & & & & & & $1087.66,1111.65$ & $18: 1(11 \Delta)$ & \\
\hline
\end{tabular}




\section{PA1}

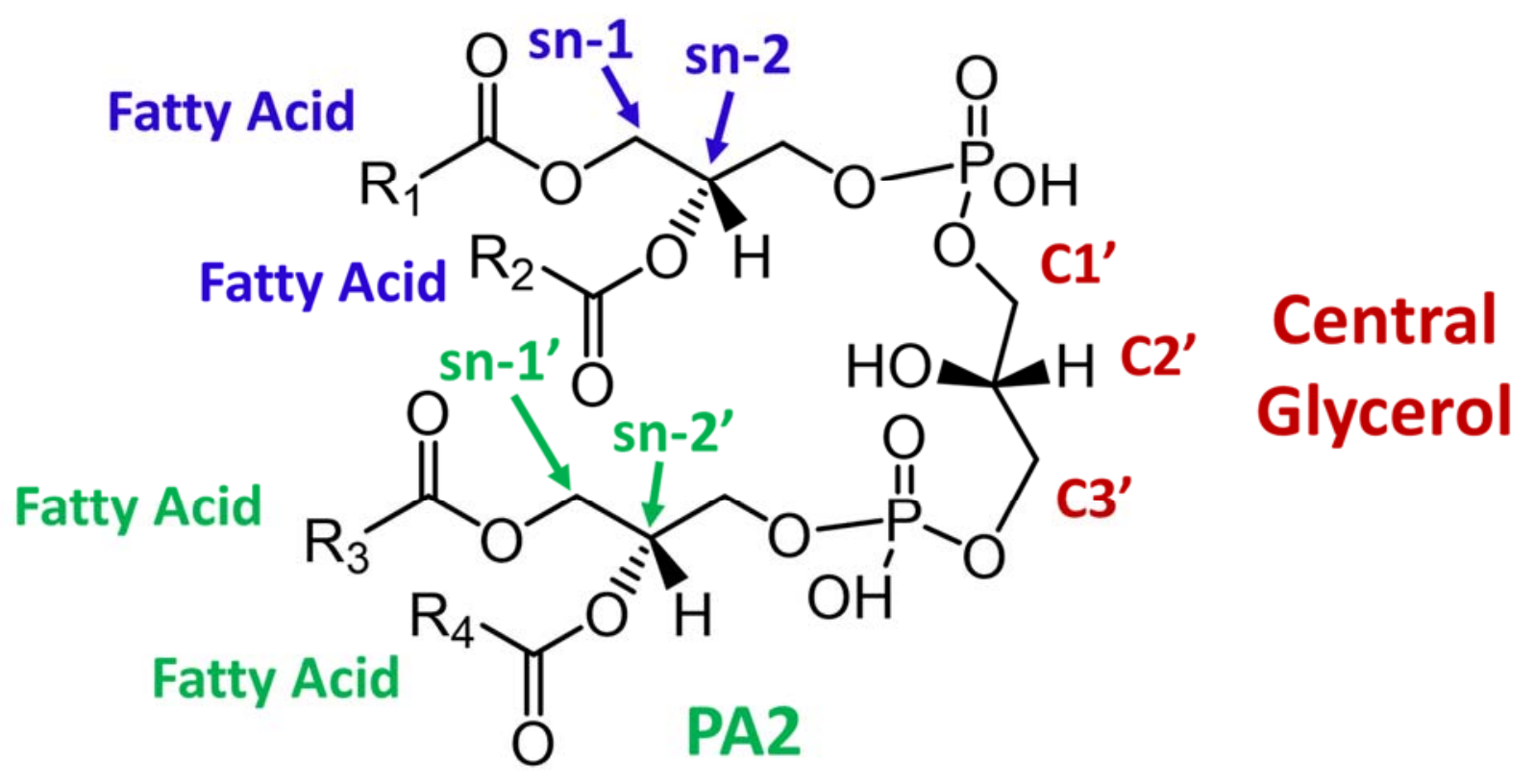

Scheme S1: Structural composition of CLs 


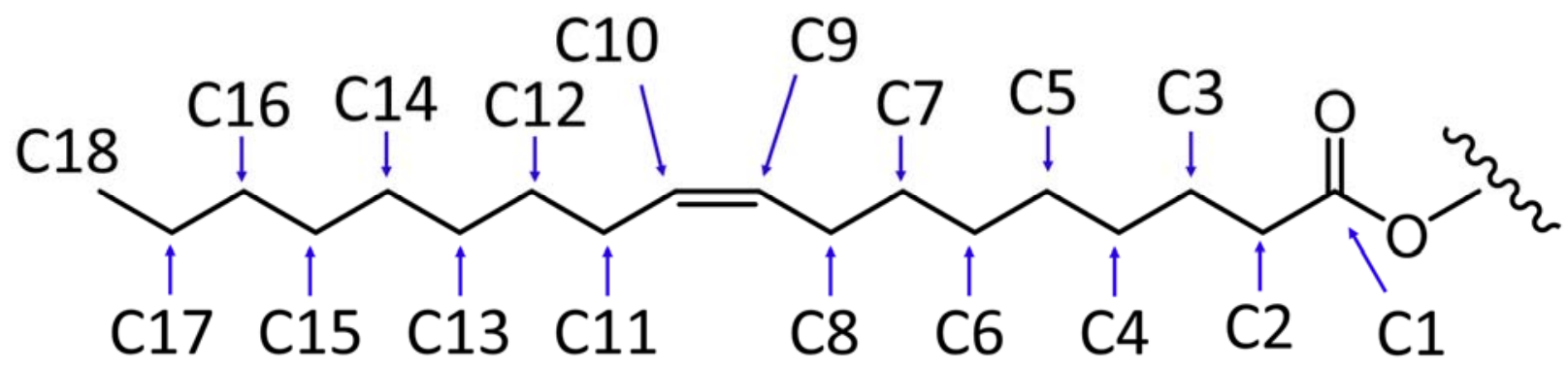

Scheme S2: Carbon numbering for fatty acids. 


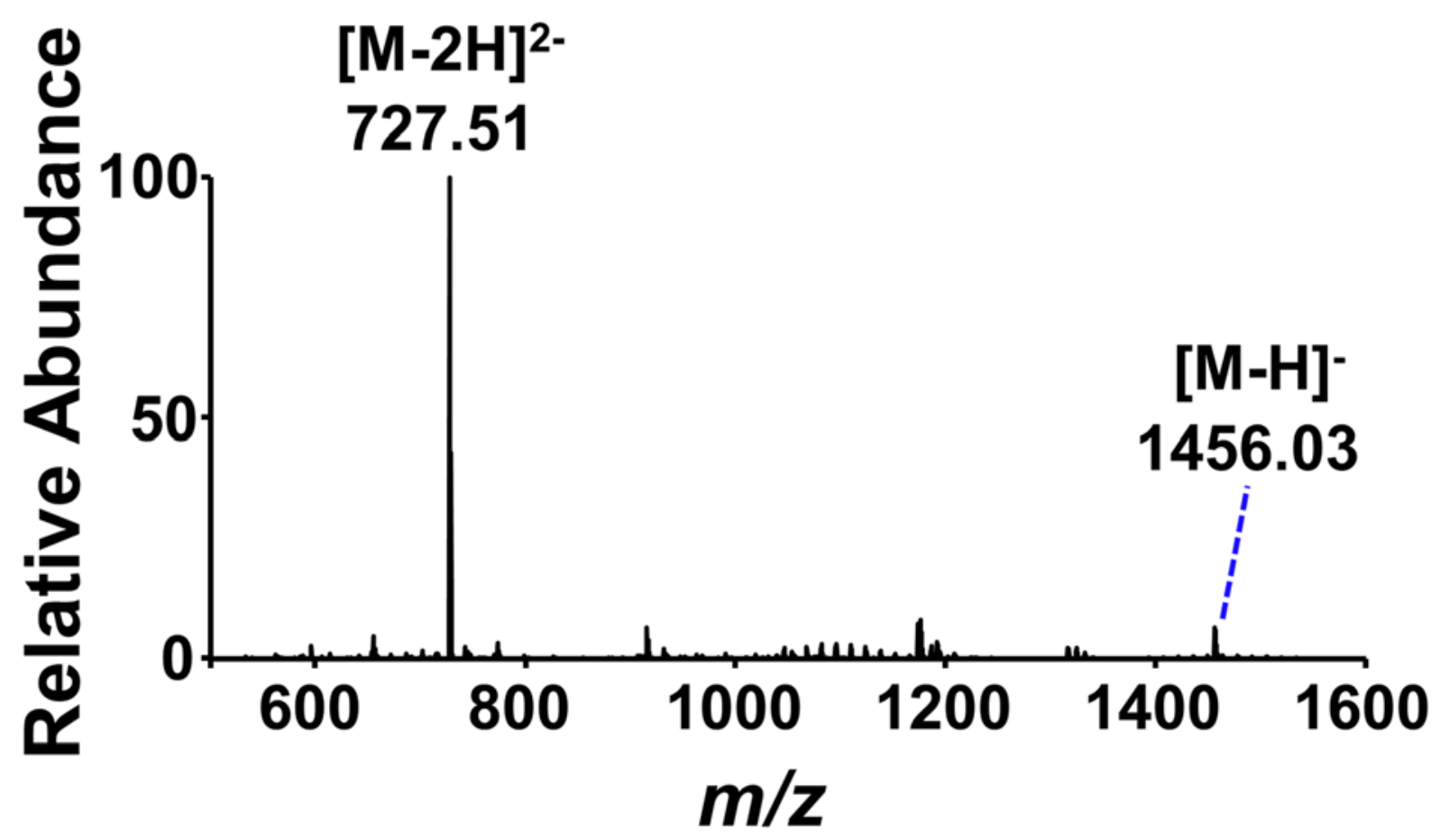

Figure S1: Negative mode $\mathrm{MS}^{1}$ spectrum of CL $(18: 1(9 \mathrm{Z}) / 18: 1(9 \mathrm{Z})) /(18: 1(9 \mathrm{Z}) / 18: 1(9 \mathrm{Z})$ (monoisotopic mass $1457.03 \mathrm{Da}$ ). $\mathrm{MS}^{2} \mathrm{CID}$ and UVPD spectra for $\mathrm{m} / \mathrm{z} 1456.03$ are shown in Figure S2 and S3, respectively. MS ${ }^{2}$ CID and UVPD spectra for $m / z 727.51$ are shown in Figure 1A and 1B, respectively. CID/UVPD for the deacylated product $(\mathrm{m} / \mathrm{z} 1173.77)$ generated via CID of $m / z 727.51$ is shown in Figure 1C. 

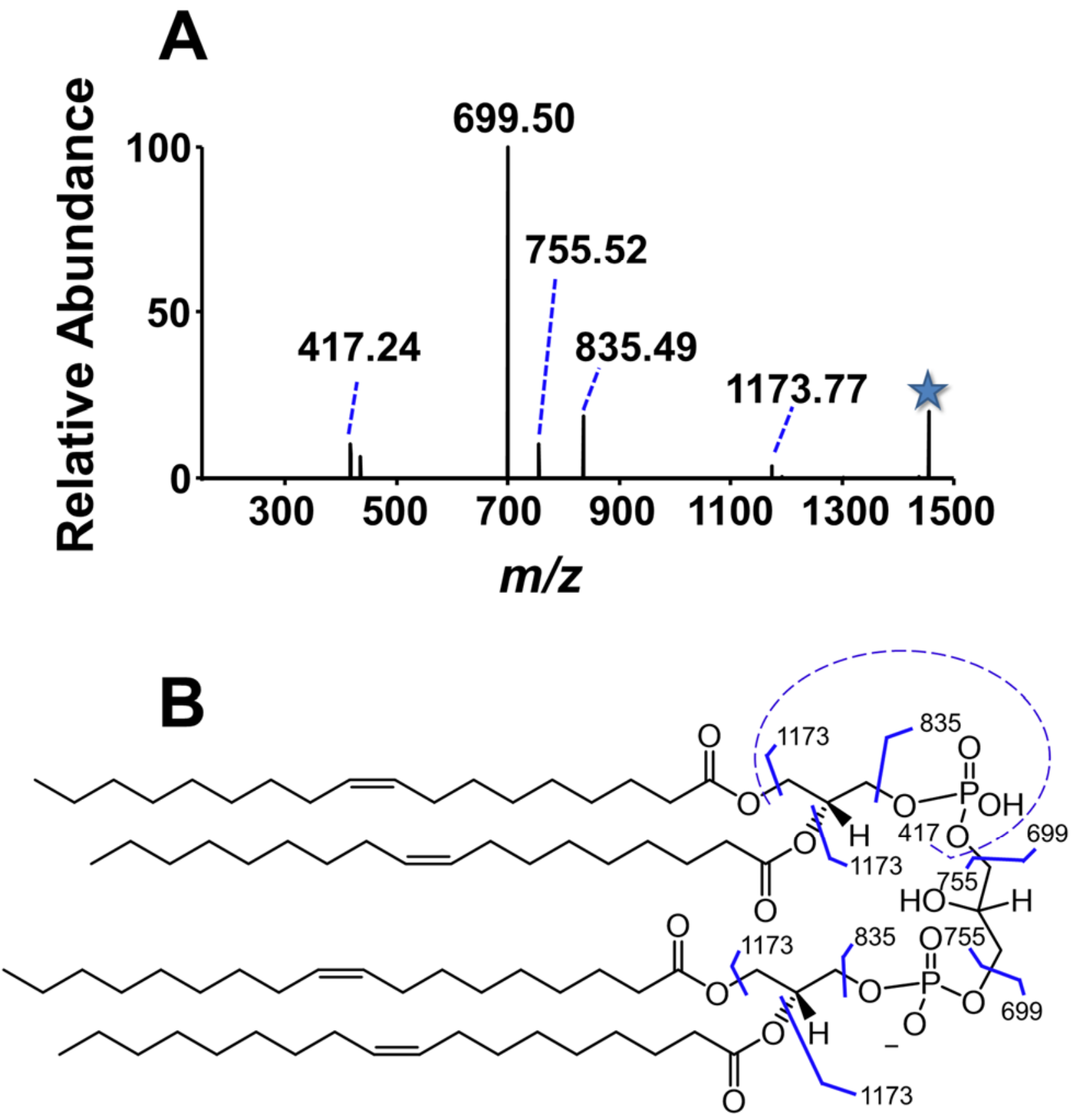

Figure S2: (A) CID (NCE 35) and (B) fragmentation map of singly deprotonated CL $(18: 1(9 Z) / 18: 1(9 Z)) /(18: 1(9 Z) / 18: 1(9 Z)(m / z ~ 1456.03)$. The selected precursor ion is designated with a star. 

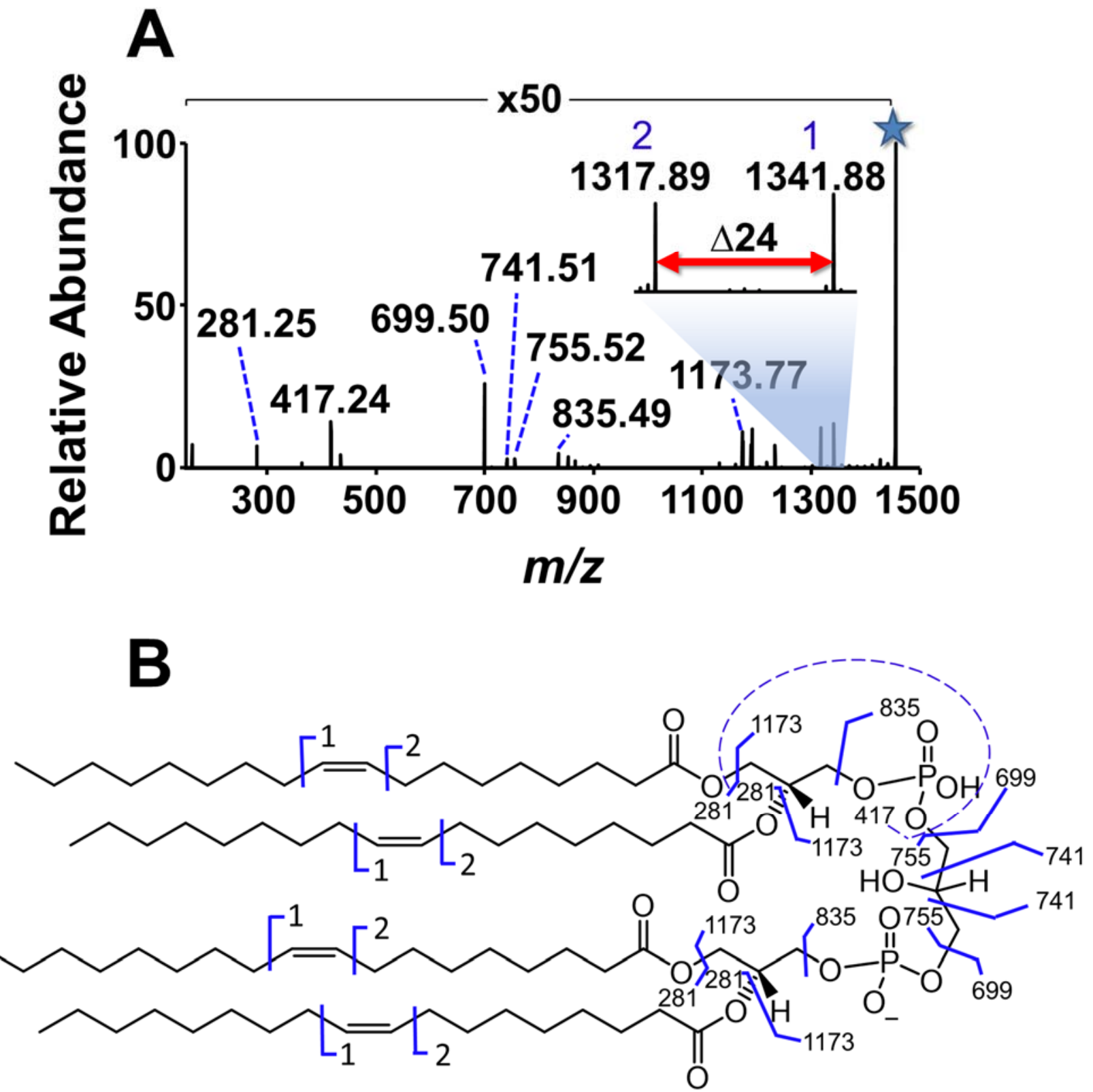

Figure S3: (A) UVPD (10 pulses, $1.0 \mathrm{~mJ}$ ) and (B) fragmentation map of singly deprotonated CL $(18: 1(9 Z) / 18: 1(9 Z)) /(18: 1(9 Z) / 18: 1(9 Z))(m / z ~ 1456.03)$. The selected precursor ion is designated with a star. 

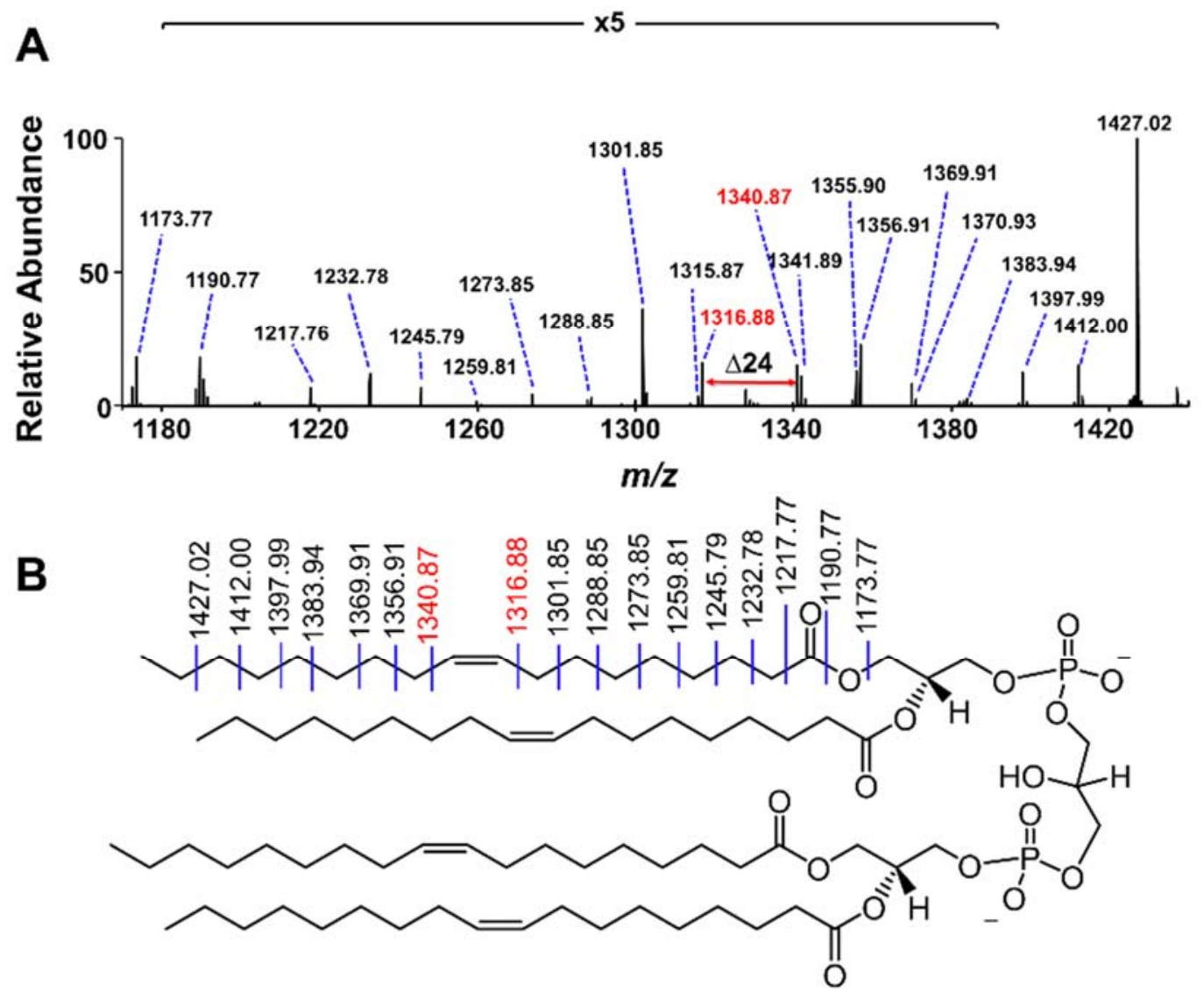

Figure S4: (A) Expanded region view of Figure 1B (UVPD spectrum for doubly deprotonated CL (18:1(9Z)/18:1(9Z))/(18:1(9Z)/18:1(9Z))) displaying the array of products originating from cleavages of $\mathrm{C}-\mathrm{C}$ bonds. Products diagnostic for the double bond locations are marked in red font (B) Product $\mathrm{m} / \mathrm{z}$ for each C-C was mapped onto one of the CL fatty acids. 


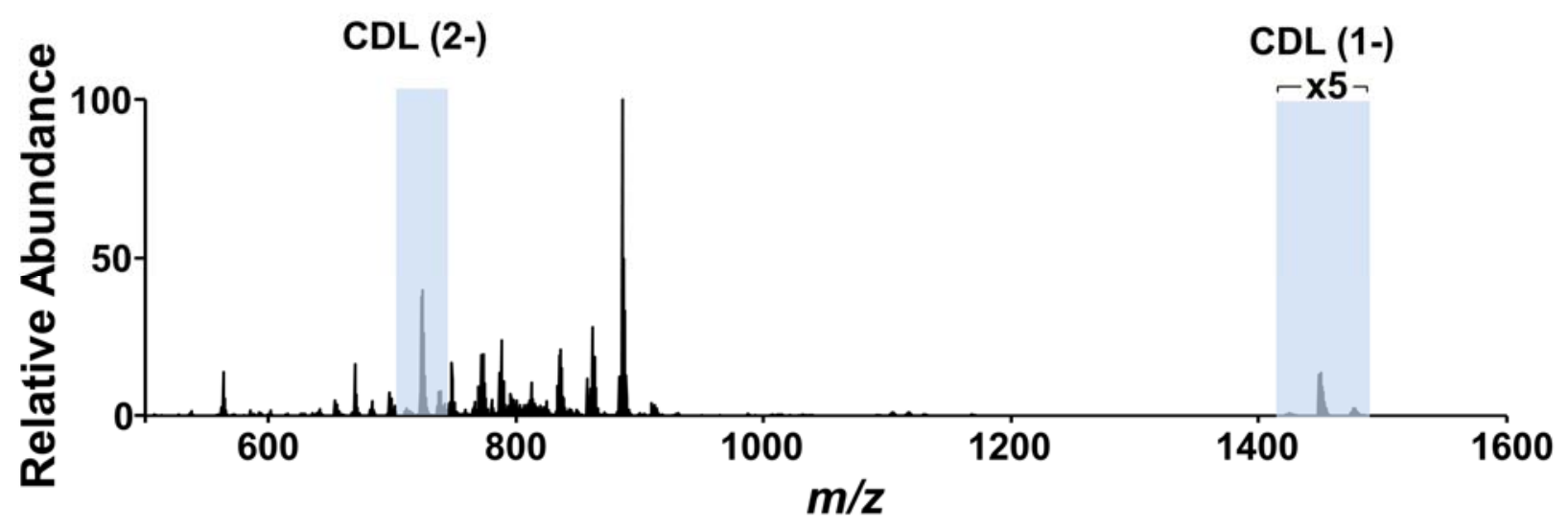

Figure S5: Negative mode $\mathrm{MS}^{1}$ spectrum of papillary thyroid carcinoma total lipid extract. 


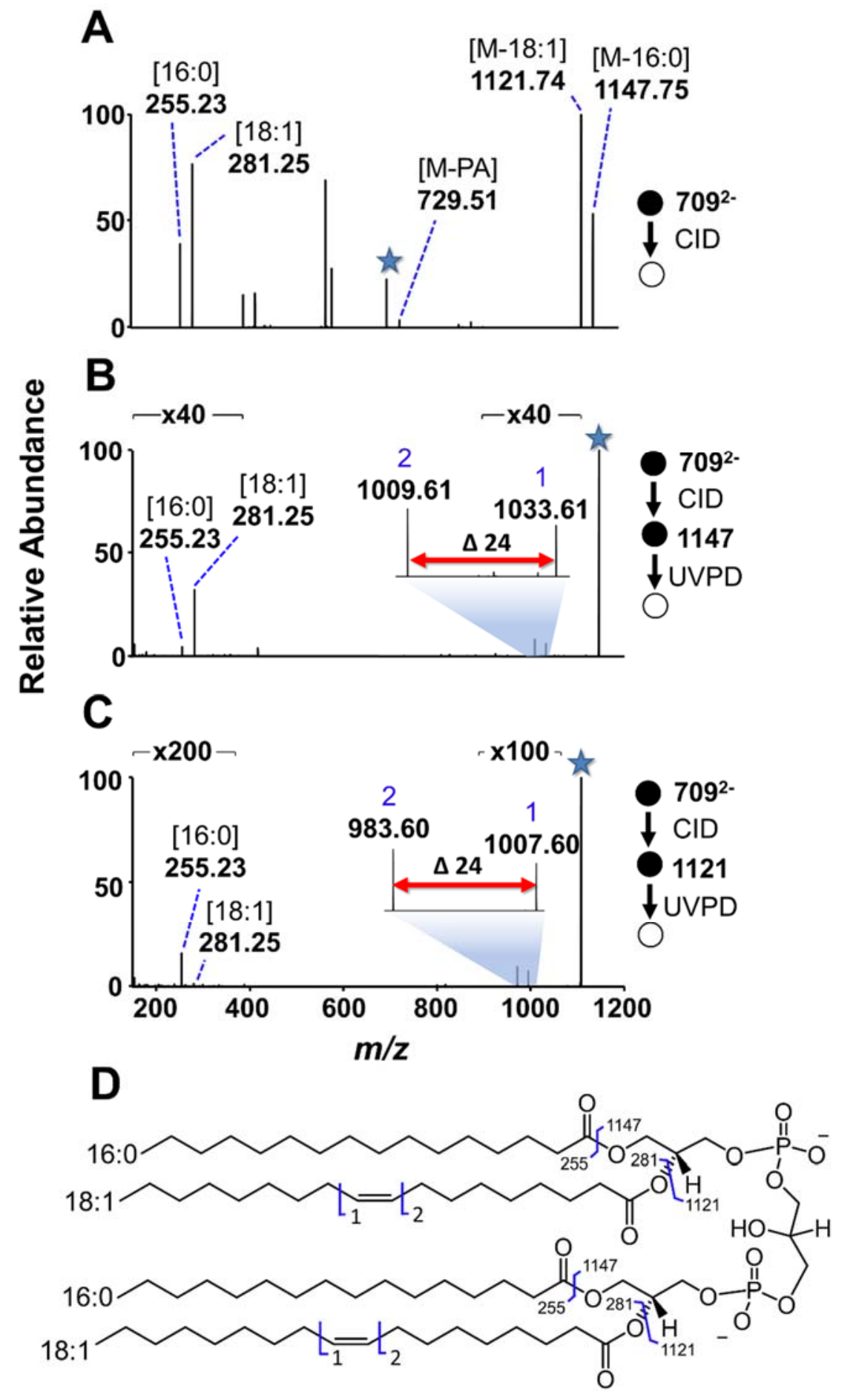

Figure S6: (A) CID of doubly deprotonated CL (16:0/18:1(9Z))/(16:0/18:1(9Z)) generates abundant deacylated products of $\mathrm{m} / \mathrm{z} 1147.75$ and $\mathrm{m} / \mathrm{z} 1121.74$ generated from the loss of a 16:0 or 18:1 fatty acid, respectively. UVPD of the ion of (B) $\mathrm{m} / \mathrm{z} 1147.75$ or (C) $\mathrm{m} / \mathrm{z} 1121.74$ produces diagnostic fragment ions that localize the double bond. (D) Fragmentation map for the CL standard. 


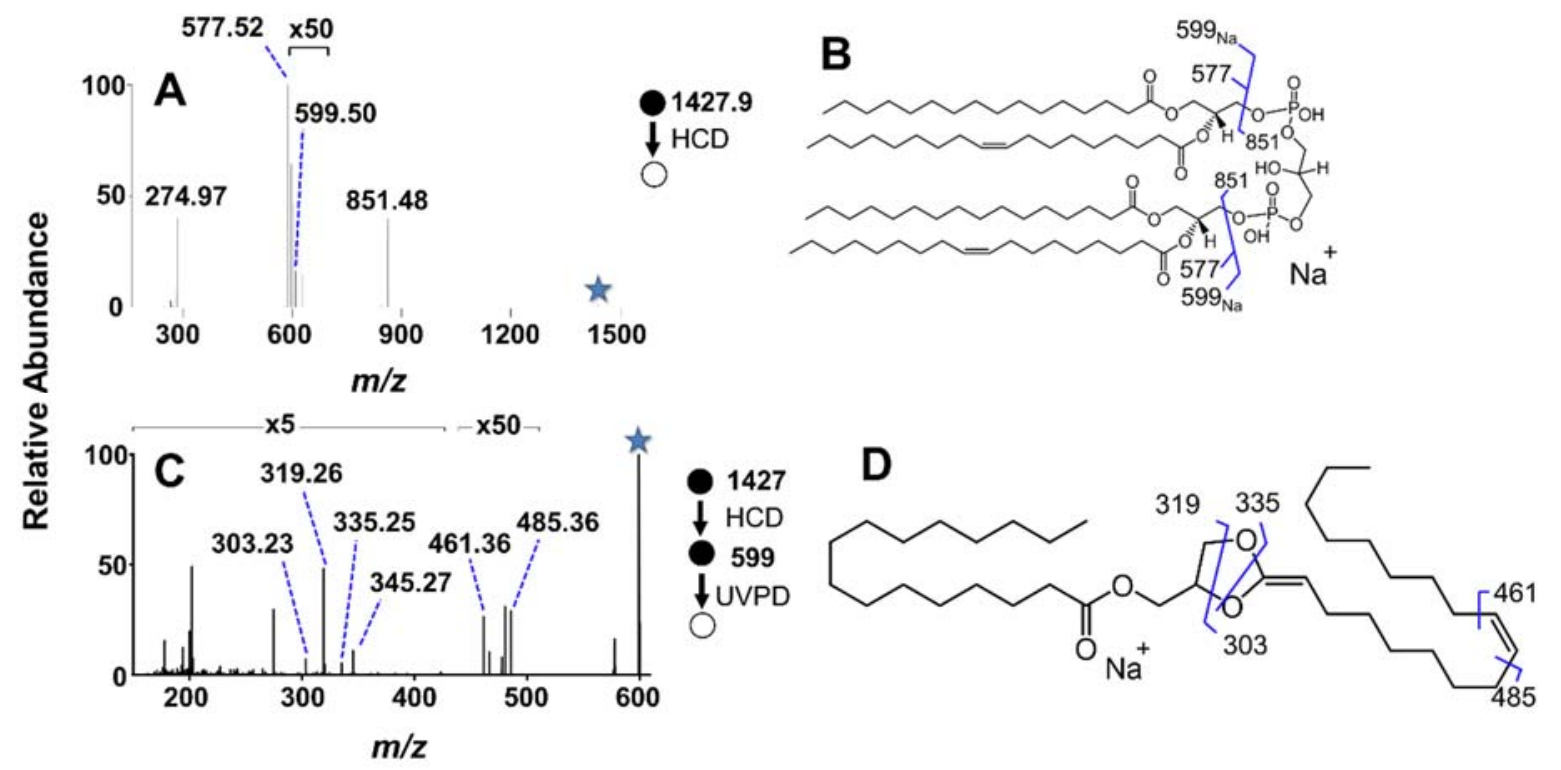

Figure S7: (A) HCD and (C) HCD/UVPD mass spectra for sodium-cationized CDL $(16: 0 / 18: 1(9 \mathrm{Z})) /(16: 0 / 18: 1(9 \mathrm{Z}))(1+), \mathrm{m} / \mathrm{z} 1427$ and corresponding fragment maps (B) and (D), respectively. HCD generates the sodium-cationized "headgroup loss"-like product of $m / z 599.50$. UVPD of the fragment ion of $\mathrm{m} / \mathrm{z} 599.50$ generated via HCD produces diagnostic fragment ions that identify the acyl chain $s n$ position. Fragment ions of $m / z 319.26$ and $m / z 335.25$ indicate the 16:0 chain is located at the $s n-1$ or $s n-1$ ' position. The ion of $m / z$ zo3.23 indicates $18: 1$ is located at the $s n-2$ or $s n-2$ ' position. The ion of $m / z 345.27$ indicates the presence of a minor isomer with one 18:1 chain at the $s n-1$ or $s n-1$ ' position. Diagnostic ions $(\mathrm{m} / \mathrm{z} 461 / 485)$ that reveal double bond locations are also produced. The selected precursor ion is designated with a star. 
A

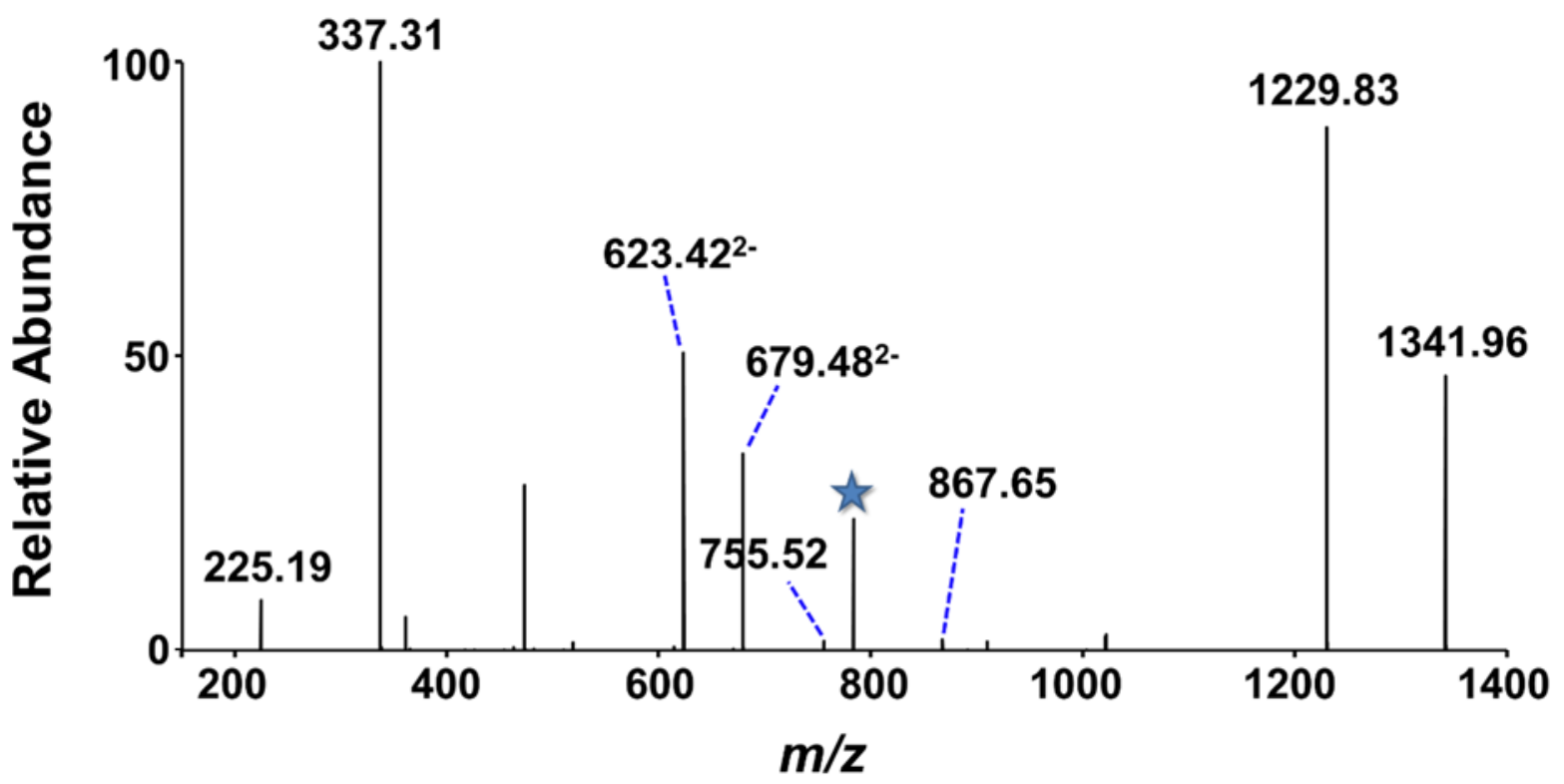

B

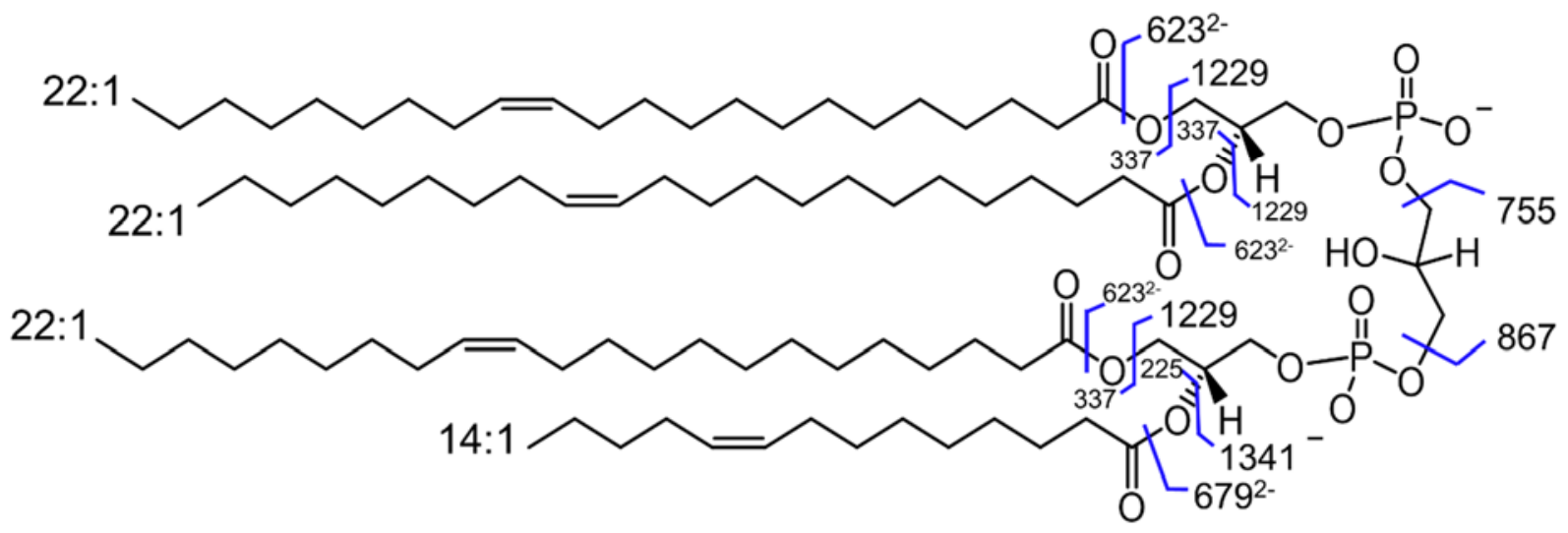

Figure S8: (A) CID (NCE 25) and (B) fragmentation map of doubly deprotonated CL $(22: 1(13 Z) / 22: 1(13 Z)) /(22: 1(13 Z) / 14: 1(9 Z)(m / z ~ 783.57)$. The selected precursor ion is designated with a star. MS ${ }^{3}$ UVPD spectra for products $m / z 1229.83$ and $\mathrm{m} / \mathrm{z} 1341.96$ are shown in Figure 2A and $2 \mathrm{~B}$, respectively. 


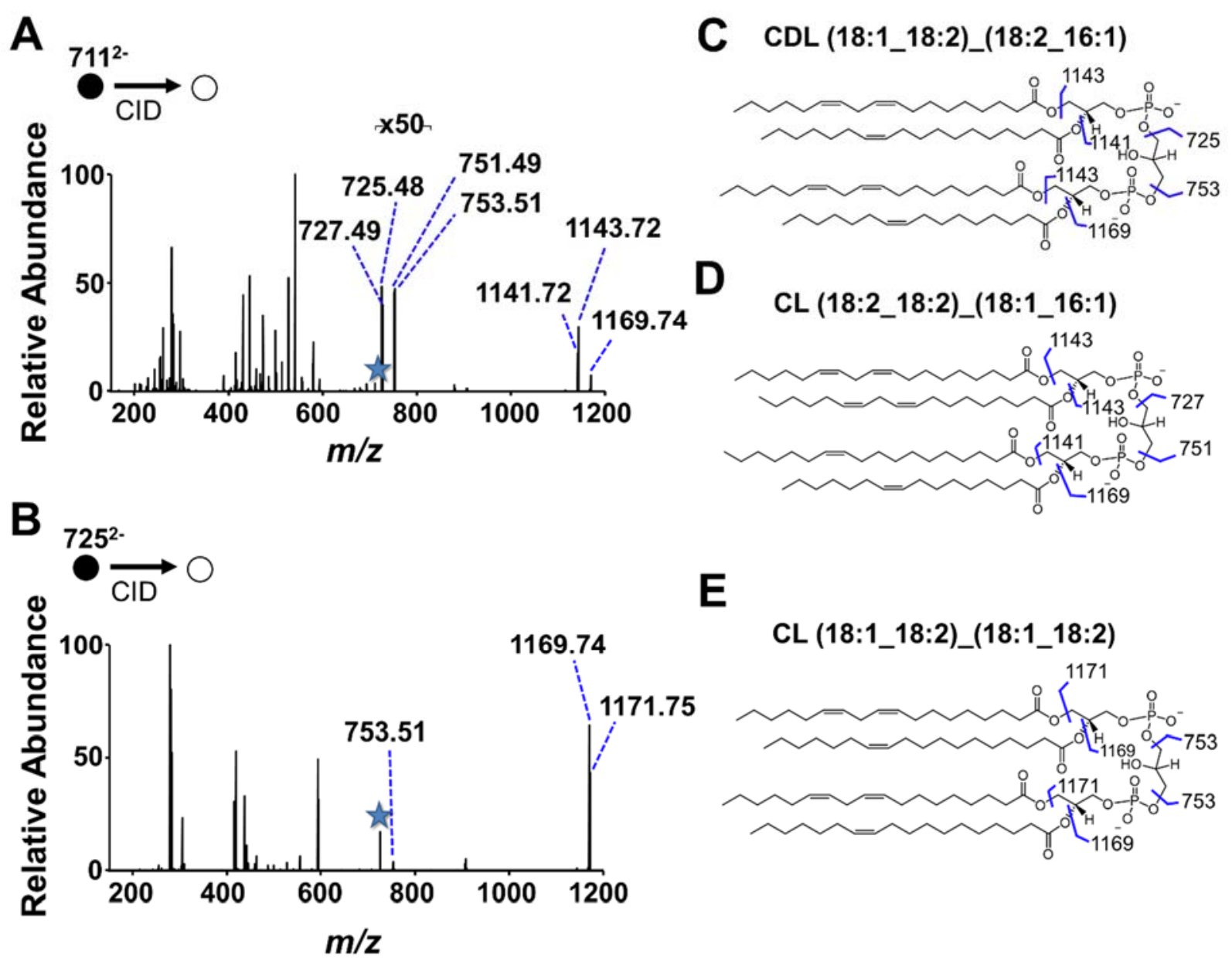

Figure S9: (A,B) CID mass spectra of $m / z 711.48$ and $m / z 725.49$ from a papillary thyroid carcinoma lipid extract, and $(\mathrm{C}, \mathrm{D}, \mathrm{E})$ assigned structures. The selected precursor ions are designated with a star. CID/UVPD of any of the deacylated fragments does not produce diagnostic ions that unambiguously localize double bond positions. 


\section{References}

(1) Liebisch, G.; Vizcaíno, J. A.; Köfeler, H.; Trötzmüller, M.; Griffiths, W. J.; Schmitz, G.; Spener, F.; Wakelam, M. J. O. Shorthand Notation for Lipid Structures Derived from Mass Spectrometry. J Lipid Res 2013, 54, 1523-1530.

(2) Blevins, M. S.; Klein, D. R.; Brodbelt, J. S. Localization of Cyclopropane Modifications in Bacterial Lipids via 213 Nm Ultraviolet Photodissociation Mass Spectrometry. Analytical Chemistry 2019. 